\title{
Toy or Tool? Utilitaristischer und hedonischer Nutzen mobiler Augmented Reality-Apps
}

\author{
Stefan Hoffmann $(\mathbb{D} \cdot$ Robert Mai $\cdot$ Timo Pagel
}

Eingegangen: 30. Juni 2021 / Angenommen: 17. November 2021 / Online publiziert: 9. Dezember 2021 (C) Der/die Autor(en) 2021

Zusammenfassung Mobile Augmented Reality (MAR)-Apps für private Konsument:innen existieren zwischenzeitlich für verschiedene Anwendungsbereiche und sie können zahlreiche Funktionen erfüllen. Der vorliegende Beitrag untersucht, welche Faktoren den wahrgenommenen Nutzen und die Bewertung von Konsument:innen beeinflussen. Hierzu werden 100 MAR-Apps anhand der Kategorien Information Layer, Unterhaltung, Bildung, virtuelle Produktproben und sonstige Hilfestellungen systematisiert und hinsichtlich ihres utilitaristischen und hedonischen Nutzens beurteilt. Während der hedonische Nutzen bei MAR-Apps der Kategorie Unterhaltung im Vordergrund steht, zielen MAR-Apps der Kategorien hilfreiche Tools und Information Layer hauptsächlich auf den funktionalen Nutzen ab. Für MAR-Apps der Kategorien Bildung und virtuelle Produktproben sind hedonische und utilitaristische Komponenten gleichermaßen wichtig. Die Untersuchung belegt, dass die von Konsument:innen auf App-Stores abgegebenen Ratings der MARApps zwar von diesen hedonischen und utilitaristischen Nutzenkomponenten getrieben werden. Allerdings zeigt sich eine wichtige Interaktion. Der hedonische Nutzen wirkt sich insbesondere dann auf die Nutzerbewertungen aus, wenn der utilitaristische Nutzen gering ist. Aus den Befunden leiten sich wichtige Implikationen für App-Entwickler und Unternehmen ab.

\footnotetext{
Stefan Hoffmann $(\bowtie) \cdot$ Timo Pagel

Institute of Business Administration, Department of Marketing, Kiel University,

Westring 425, 24098 Kiel, Deutschland

E-Mail: stefan.hoffmann@bwl.uni-kiel.de

Timo Pagel

E-Mail: stu202867@mail.uni-kiel.de

Robert Mai

Department of Marketing, Grenoble Ecole de Management, 12 Rue Pierre Semard, 38000 Grenoble,

Frankreich

E-Mail: robert.mai@grenoble-em.com
} 
Schlüsselwörter Augmented Reality · Apps · Utilitaristisch · Hedonisch · User Rating · Mobile Augmented Reality (MAR)

\title{
Toy or Tool? Utilitarian and Hedonic Benefit of Mobile Augmented Reality Apps
}

\begin{abstract}
Mobile Augmented Reality (MAR) apps now exist for various applications and can fulfill numerous functions for private consumers. This paper investigates the factors that influence consumers' perceived benefits and the evaluation of MAR apps. For this purpose, 100 MAR apps are systematized according to the categories information layer, entertainment, education, virtual product samples, and other assistance. These apps are evaluated with respect to their utilitarian and hedonic benefits. While hedonic benefit is particularly relevant for MAR apps in the entertainment category, MAR apps in the categories helpful tools and information layer mainly target functional benefit. For MAR apps in the categories education and virtual product samples, hedonic and utilitarian components are equally important. The study confirms that actual user ratings of MAR apps given on app stores are indeed driven by these hedonic and utilitarian benefit components. However, an important interaction emerges. Hedonic benefits affect user ratings especially when utilitarian benefits are low. Important implications for app developers and companies are derived from the findings.
\end{abstract}

Keywords Augmented Reality · Apps · Utilitarian · Hedonic · User Rating · Mobile Augmented Reality (MAR)

\section{Einleitung}

Augmented-Reality (AR) erzeugt auf einem Endgerät ein Abbild der realen Welt, welches durch virtuelle Elemente erweitert wird. Eine Person, ein Objekt oder die natürliche Umgebung wird in Echtzeit mit virtuellen Objekten überlagert, sodass bei Nutzer:innen der Eindruck entsteht, dass diese koexistieren (Azuma et al. 2001). Augmented Reality zeichnet sich zudem dadurch aus, dass Nutzer:innen auch mit den virtuellen Elementen interagieren können (Zhou et al. 2008). Angesichts der vielfältigen Einsatzmöglichkeiten verwundert es nicht, dass der AR-Technologie eine immer rasantere Diffusion vorhergesagt wird. Mehrere aktuelle Prognosereports stufen AR als einen der Top-10-Technologietrends ein (Samsung Business Insights 2020; Forbes 2020). Ein Bericht von vXchange (2020) sagt voraus, dass der ARMarkt bis 2023 einen Umsatz von 75 Mrd. US-Dollar erreichen wird. Und ein Bericht von VynZ Research (2020) prognostiziert, dass der globale AR- und Virtual Reality (VR)-Markt bis 2025 einen Umsatz von 161,1 Mrd. US-Dollar erlangt.

Dass die AR-Technologie zukünftig auch mehr und mehr von privaten Konsument:innen genutzt werden wird, wird dadurch vorangetrieben, dass Smartphones und Tablet-Computer AR-fähige Endgeräte darstellen, die zwischenzeitlich nahezu omnipräsent und in den Alltag der meisten Konsument:innen integriert sind. Konsument:innen können sich bereits jetzt zahlreiche AR-Apps auf ihren mobilen 
Endgeräten installieren und diese in unterschiedlichen Kontexten und für verschiedene Funktionen nutzen. Diese Einsatzmöglichkeiten der AR-Technologie können als Mobile Augmented Reality (MAR)-Apps bezeichnet werden (Aluri 2017; Dacko 2017; Hamari et al. 2019; Olsson et al. 2013; Rauschnabel et al. 2019). MAR-Apps für private Konsument:innen können der Unterhaltung dienen oder der Weiterbildung, sie können virtuelle Produktproben ermöglichen, Hilfestellung für Spezialaufgaben bieten oder mit einer virtuellen Informationsschicht Objekte überlagern, um Nutzer:innen tiefergehend zu informieren.

Doch warum nutzen Konsument:innen MAR-Apps auf ihren privaten Geräten? Bekannte Beispiele wie Pokémon Go lassen vermuten, dass bei der Nutzung von MAR insbesondere positive Erlebnisse, Spaß und Zerstreuung im Vordergrund stehen (Hamari et al. 2019; Rauschnabel et al. 2017; Yang und Liu 2017). MARApps werden somit als „Toy“, als Spielzeug, eingesetzt und der hedonische Nutzen scheint zu dominieren (Olsson et al. 2013). Bildungsangebote oder die Übermittlung von Produktinformationen via MAR lassen jedoch auch utilitaristische Funktionen erkennen (Dacko 2017; Joerß et al. 2021). MAR wird hierbei zum „Tool“, zum Werkzeug, das Konsument:innen in ihrem Alltag unterstützen soll. Der vorliegende Beitrag klärt die Frage, inwiefern MAR von privaten Konsument:innen als Toy oder als Tool angesehen wird und welche Einsatzpotenziale sich daraus ergeben.

Die vorliegende Studie analysiert 100 MAR-Apps, die auf Plattformen wie Google Play und Apple-Store zum Download zur Verfügung stehen, um den Beitrag hedonischer und utilitaristischer Nutzenkomponenten auf die Bewertung von MARApps zu identifizieren. Hierbei wird der jeweilige Beitrag dieser Komponenten bei verschiedenen Kategorien von MAR-Apps differenziert betrachtet. Zudem deckt die Untersuchung auf, inwiefern die utilitaristische und hedonische Nutzenkomponenten interagieren.

\section{Augmented Reality}

\subsection{Begriffsbestimmung und Abgrenzung}

Augmented-Reality (AR) sollte von verwandten Technologien wie Virtual Reality (VR) abgegrenzt werden. AR erzeugt in Echtzeit ein digitales Abbild der physischen Umgebung auf einem Bildschirm, das durch computergenerierte Elemente erweitert wird, mit denen Nutzer:innen interagieren können (Azuma et al. 2001; Zhou et al. 2008). Auch VR kreiert eine digitale Umgebung, in der Nutzer:innen in Echtzeit mit den virtuellen Objekten interagieren können. Allerdings schafft VR eine vollständig computergenerierte Welt, während AR die reale Welt mit computergenerierten Objekten überlagert (Flavián et al. 2019) und die Interaktion somit in einer Umgebung stattfindet, in der die physische und digitale Welt miteinander verschmolzen sind. Um diese Unterschiede zu visualisieren, stellt das Reality-Virtuality-Kontinuum von Milgram und Kishino (1994) die reale Umgebung an das eine Ende des Kontinuums und die vollständige Virtualität an das gegenüber liegende Ende. Der Bereich zwischen diesen Extremen wird als gemischte Realität bezeichnet. Augmented Reality beschreibt, dass die reale Umgebung durch virtuelle Elemente erweitert wird. 
Die Augmentation der Realität durch virtuelle Elemente kann auf verschiedenen Displays und Geräten realisiert werden. Erstens können stationäre Bildschirme wie Computermonitore und Laptops genutzt werden. Ein interessantes Anwendungsbeispiel hierfür sind virtuelle Try-Ons, d.h. Tools, die Konsument:innen ermöglichen Produkte (insb. Schmuck, Brillen, Bekleidung, Schuhe, Kosmetik etc.) virtuell anzuprobieren, indem die mit einer Kamera gefilmte Person in Echtzeit ganz oder teilweise auf einem Monitor gezeigt und mit den zu probierenden Produkten überlagert wird. In diese Kategorie fallen u.a. virtuelle Spiegel (virtual mirror oder magic mirror), die beispielsweise im Bekleidungseinzelhandel auf Monitoren oder im E-Commerce über den Bildschirm eines privaten PCs oder Laptops eingesetzt werden können. Zweitens können Head-Up-Displays (HUD) genutzt werden, wenn Nutzer:innen eine bestimmte Kopfhaltung und Blickrichtung einnehmen, wie beispielsweise beim Autofahren, wo Navigationshinweise auf die Windschutzscheibe projiziert werden können und die Informationen damit direkt im Sichtfeld erscheinen. Drittens können tragbare mobile Geräte wie Smartphones, Smartwatches oder Tablet-Computer genutzt werden (Brito und Stoyanova 2018; Carmigniani et al. 2011; Kim und Hyun 2016). Viertens können auch Wearable-Technologien eingesetzt werden. Dazu gehören Head-Mounted-Displays wie smarte Brillen oder Helme (z.B. Microsoft HoloLens), die das Sichtfeld der Nutzer:innen mit digitalen Objekten überlagern (z. B. Brito und Stoyanova 2018; Rauschnabel 2018; Rauschnabel et al. 2018). Fünftens können zukünftig möglicherweise auch verstärkt implantierte Geräte, wie AR-fähige Kontaktlinsen, genutzt werden (Flavián et al. 2019). Für private Nutzer:innen sind derzeit jedoch mobile Geräte aufgrund der weiten Verbreitung sicherlich der interessanteste Anwendungsfall. Der vorliegende Artikel fokussiert deshalb auf diese Anwendung.

\subsection{Einsatzgebiete von MAR-Apps}

Das bekannteste Beispiel für den Einsatz von MAR für Konsument:innen ist das Spiel Pokémon Go, bei dem die Spieler:innen nach versteckten virtuellen Pokémons in ihrer physischen Umgebung suchen (Hamari et al. 2019; Rauschnabel et al. 2017; Yang und Liu 2017). Andere eindrucksvolle Beispiele sind MAR-Apps, die den Nachthimmel mit computergenerierten Informationen über die Gestirne und Sternbilder überlagern (z. B. Night Sky, Sky View, Star Walk). Auch im Tourismussektor wird MAR häufig eingesetzt, um ortsbasierte Informationen über Sehenswürdigkeiten bereitzustellen oder in Museen objektbasierte Informationen über Exponate zu bieten (z. B. Time Traveller, CorfuAR; Kourouthanassis et al. 2015; tom Dieck und Jung 2018). Auch Zeitschriften (z.B. AUTO BILD) konnten schon virtuell erweitert werden, um zusätzliche Informationen abzurufen (z. B. Rese et al. 2017). Andere MAR-Apps übersetzen Sprachen in Echtzeit und helfen somit, beispielsweise Hinweisschilder, Speisekarten etc. in fremden Sprachen zu lesen. Manche MAR-Applikationen bieten die Möglichkeit, Möbel oder Wandfarbe in der eigenen Wohnung virtuell zu testen (z. B. IKEA place, Cimagine; z. B. Rese et al. 2017). Besonders weite Verbreitung fanden MAR-Applikationen auch schon, um Kleidung, Schuhe, Uhren, (Sonnen-)Brillen und weitere Accessoires (Ray Ban Virtual Try- 
On, Mr. Spex) oder Kosmetik (Shisedio AR Makeup Mirror) virtuell zu testen (z. B. Huang und Liao 2015; Rese et al. 2017; Yim et al. 2017).

\subsection{Fokus der AR-Studien in der Konsumentenforschung}

Der vorliegende Beitrag betrachtet MAR-Apps aus der Perspektive der Konsumentenforschung, die hier zunächst eingeordnet werden soll. Neben der Informatik, die sich unter anderem mit den technischen und funktionalen Aspekten der ARTechnologie, wie bspw. der Objektpositionierung oder Steuerung beschäftigt (Chae et al. 2018; Carmigniani et al. 2011; Kytö et al. 2018; Zhou et al. 2008), widmen sich auch zahlreiche andere Disziplinen der AR-Technologie, wobei hier meist die Anwendungs- und Einsatzgebiete im Vordergrund stehen. Hierzu zählen beispielsweise die Bildungswissenschaften (Bower et al. 2014; Di Serio et al. 2013; Harley et al. 2016), Psychologie (Botella et al. 2005), Medizin (Berryman 2012; Vávra et al. 2017), Gaming (Hamari et al. 2019; Rauschnabel et al. 2017) oder Tourismusforschung (Aluri 2017; Chung et al. 2018; tom Dieck und Jung 2018). In der Management-Literatur wurde AR unter anderem mit Fokus auf Produktion und Industrie 4.0 (Kaasinen et al. 2020; Masood und Egger 2019) untersucht. Die Marketingwissenschaften betrachten den Einsatz für Werbung und Branding (Hopp und Gangadharbatla 2016; Yaoyuneyong et al. 2016). Das Handelsmanagement untersucht den Einsatz für e-Commerce und m-Commerce (z. B. Baek et al. 2018; Beck und Crié 2018; Javornik 2016), aber auch für den stationären Handel (z. B. Joerß et al. 2021).

Studien, die sich mit der Perspektive der Konsument:innen beschäftigen, betrachten als Zielvariablen unter anderem die (Wieder-)Nutzungsintention (z. B. Pantano et al. 2017; Rese et al. 2017; Spreer und Kallweit 2014), Kaufabsicht (z. B. Hilken et al. 2017; Yim et al. 2017) und Weiterempfehlungsabsichten (z. B. Hilken et al. 2017). Die Konsumentenforschung hat in den letzten Jahren zahlreiche Einflussgrößen ermittelt, die diese Zielvariablen beeinflussen können. Dazu zählen die Art der Augmentation (Heller et al. 2019a; Javornik 2016), die Interaktionsqualität (Yim et al. 2017; Heller et al. 2019b), Vorbehalte der Konsumenten gegenüber der Technologie (Rauschnabel et al. 2018), die User Experience (Javornik 2016; Rese et al. 2017), die Technologieakzeptanz (Pantano et al. 2017; Rese et al. 2017) und nicht zuletzt der erwartete Nutzen (McLean und Wilson 2019; Pantano et al. 2017; Rese et al. 2017; Yim et al. 2017). In dem vorliegenden Beitrag betrachten wir insbesondere die Rolle von hedonischer und utilitaristischer Nutzenkomponenten der MARApps.

\section{Hedonische und Utilitaristische Nutzenkomponenten}

Konsumentenforscher unterscheiden zwischen utilitaristischem und hedonischem Konsumnutzen (Babin et al. 1994; Childers et al. 2001; Dhar und Wertenbroch 2000). Zu den utilitaristischen Nutzenkomponenten zählen u. a. Funktionalitäten sowie die Möglichkeit, relevante Informationen zu übermitteln (van der Heijden 2004). Hedonische Nutzenkomponenten umfassen insbesondere die Freude und den Spaß 
während der Nutzung. Hierzu zählen beispielsweise auch Aspekte wie Gamification und Ästhetik.

Als theoretische Grundlage für die Übertragung dieser Nutzenkomponenten auf die AR-Technologie dient dabei insbesondere das Technology Acceptance Model (TAM) von Davis (1989) sowie dessen Weiterentwicklungen (z. B. UTAUT, UTAUT2; Venkatesh et al. 2003) die bereits in zahlreichen AR-Studien Anwendung fanden (z. B. Huang und Liao 2015; McLean und Wilson 2019; Pantano et al. 2017; Plotkina und Saurel 2019; Rese et al. 2017; Rauschnabel et al. 2018; Spreer und Kallweit 2014). Das Konstrukt wahrgenommene Nützlichkeit (,Perceived Usefulness“) spiegelt dabei die utilitaristische Komponente wider, während die hedonische Komponente durch wahrgenommenes Vergnügen (,Perceived Enjoyment“) abgebildet wird. Auch der Uses-and-Gratification-Approach (Ruggiero 2000) kann als weitere theoretische Grundlage herangezogen werden (z. B. Rauschnabel 2018; Rauschnabel et al. 2018; Yang und Liu 2017).

Es liegen bereits einige Studien vor, welche die Bedeutung dieser beiden Komponenten erfassten. Bei Unterhaltungsmedien wurde bislang vor allem die hedonische Komponente bestätigt. Beispielsweise zeigen Rauschnabel et al. (2017), Yang und Liu (2017) wie auch Hamari et al. (2019) am Beispiel von Pokémon Go, dass bei MAR-Games Spaß und Freude wichtige Treiber sind. Studien, die sich mit dem Einsatz von MAR als Information Layer am Point-of-Sale beschäftigten, zeigen auch die Bedeutung der utilitaristischen Komponente auf. So belegt die Studie von Spreer und Kallweit (2014), dass neben dem wahrgenommenen Vergnügen auch die wahrgenommene Nützlichkeit positive Effekte hat. Studien zu virtuellen Produktproben attestieren positive Effekte zumeist für utilitaristische (z. B. wahrgenommene Nützlichkeit, wahrgenommene Informiertheit), aber auch für hedonische (z. B. wahrgenommenes Vergnügen, wahrgenommene Verspieltheit, wahrgenommene Ästhetik) Nutzenkomponenten. Studien belegen dies beispielsweise für virtuelle Produktproben von Bekleidung (Huang und Liao 2015; Plotkina und Saurel 2019), Kosmetik (Smink et al. 2019) und (Sonnen-)Brillen (Pantano et al. 2017; Rese et al. 2017; Yim et al. 2017), aber auch für Möbelplaner (Rauschnabel et al. 2019; Rese et al. 2017) und andere Retailing-MAR (z. B. McLean und Wilson 2019).

Die genannten Studien fokussieren auf einzelne MAR-Anwendungsfälle und basieren auf Selbstauskünften von speziell für diese Zwecke Befragten. Die vorliegende Studie soll unabhängig von einer Untersuchungssituation abgegebene Beurteilungen vergleichend über verschiedene MAR-Anwendungen untersuchen.

\section{Untersuchungsdesign}

\subsection{Analysierte MAR-Apps}

Die Grundgesamtheit der Untersuchungsobjekte bildeten alle MAR-Apps, die zum Untersuchungszeitpunkt auf den Plattformen Google Play Store und Apple App Store verfügbar waren. Eine Vorauswahl der untersuchten Apps wurde anhand der folgenden Kriterien getroffen: Es wurden nur Apps ausgewählt, die sich über die genannten Stores beziehen lassen. MAR-Apps, die über weitere Plattformen oder 
Websites angeboten werden, wurden ausgeschlossen, um nur Apps einzuschließen, die von einer größeren Nutzer:innengruppe bezogenen werden können. Ferner wurden nur kostenfreie Apps ausgewählt, die sich an private Konsument:innen richten. MAR-Apps, die auf eine industrielle oder medizinische Anwendung abzielen, wurden aufgrund der Schwerpunktsetzung auf die Nutzung durch private Endverbraucher ausgeschlossen. Diese Applikationen werden ohnehin meist über andere Vertriebsformen und nicht über die genannten App-Stores bezogen. Als finales Einschlusskriterium mussten die untersuchten Apps funktionstüchtig sein. Da einige MAR-Apps bereits längere Zeit vor dem Untersuchungszeitraum veröffentlicht und dann nicht weiter gepflegt und aktualisiert wurden, wurden alle angebotenen Apps einer Prüfung auf Aktualität und Funktionstüchtigkeit unterzogen. Nach diesem Ausschlussverfahren entstand eine vorab definierte Stichprobe von 100 MAR-Apps, die auf den beiden relevantesten Plattformen Google Play Store $(n=75)$ und Apple App Store $(n=25)$ kostenfrei angeboten wurden.

Die am häufigsten vertretenen Kategorien nach der Zuordnung der Stores waren Entertainment, Education, Shopping, Adventure, Social Media, Tools, Art \& Design, Lifestyle, Photography, Navigation, Office und Simulation. Da diese von der AppStores vorgesehene Kategorisierung nach Themen für eine wissenschaftliche Untersuchung zu unscharf ist und sich die Einordnungen nach Anwendungsbereichen, Tätigkeiten und Funktionen überschneiden, wurde vorab eine klare Kategorisierung anhand der durch die MAR-Apps bereitgestellten Funktionen entwickelt, die aus einem möglichst kleinen Set abgrenzbarer Kategorien bestehen sollte. Die MARApps wurden anschließend nach einem vordefinierten Kodierschema den fünf so entwickelten Kategorien zugeordnet: (1) Unterhaltung und soziale Medien [ $n=41]$, (2) Bildung [11], (3) virtuelle Produktproben [26], (4) hilfreiche Tools [17] und (5) Information Layer [5].

\subsection{Bewertungskriterien}

Die Bewertung der MAR-Apps basiert auf einer Kombination objektiver Kriterien mit der Beurteilung der App durch einen Kodierer. Als objektives Maß für die Nutzerbewertung erfassten wir die User Ratings der MAR-Apps aus den App-Stores. Diese basieren auf einer Einstufung von ein bis max. fünf Sterne. Als objektive Kontrollvariablen erfassten wir auch die Anzahl der Bewertungen. Die Anzahl der Downloads floss nicht ein, da sie nur für AR-Apps verfügbar ist, die auf dem Google Play Store angeboten werden (d.h. für 75\% der untersuchten MAR-Apps). Für diese Apps liegt die Korrelation zwischen Bewertung und Downloads bei $r=0,481$ $(p \leq 0,001)$.

Alle MAR-Apps wurden von einem Kodierer hinsichtlich der erwarteten hedonischen und utilitaristischen Nutzenkomponenten eingeschätzt. Hierzu wurden alle MAR-Apps heruntergeladen, installiert, getestet und inspiziert. Für MAR-Apps, die bei Google Play angeboten wurden und die auf dem Betriebssystem Android laufen, kam ein Samsung Galaxy S9 zum Einsatz. MAR-Apps vom Apple-Store, die auf iOS laufen, wurden auf einem Apple iPhone 10s getestet. Da es in einigen Fällen Probleme mit der Funktionalität gab, wurde noch ein Apple iPad Air 9.7 herangezogen. Um zu vermeiden, dass der Kodierer einem Bias unterliegt, wurde zunächst von 
Tab. 1 Deskriptive Statistiken und Interkorrelationen

\begin{tabular}{lrrrrr}
\hline & \multicolumn{2}{l}{ M } & \multicolumn{2}{l}{ Korrelationen } & \\
& & & Hedonisch & Utilitaristisch & User Ratings \\
\hline Hedonisch & 4,87 & 2,32 & & & \\
Utilitaristisch & 4,81 & 3,53 & $-0,206^{*}$ & & \\
User Ratings & 4,07 & 0,56 & $0,269^{* *}$ & $0,385^{* * *}$ & \\
Anzahl Bewertungen & 2.238 .370 & 12.621 .790 & $0,224^{*}$ & $-0,093$ & 0,087 \\
\hline
\end{tabular}

Pearson-Produkt-Moment-Korrelationen

Signifikanzniveau: $* p \leq 0,05, * * p \leq 0,01, * * * p \leq 0,001$

zwei Versuchsleitern ein Kodierschema definiert und Kodieranweisungen entwickelt, um sicherzustellen, dass alle Apps anhand derselben Merkmale als hedonisch und utilitaristisch eingestuft wurden. Die Auswahl der Indikatoren für die Zuordnung basierte auf einschlägigen Quellen zum hedonischen und utilitaristischen Produktnutzen (Babin et al. 1994; Childers et al. 2001; Dhar und Wertenbroch 2000; van der Heijden 2004). Der Kodierer wurde erst im Anschluss daran eingewiesen. Er nahm die Zuordnung der Apps streng nach diesem Kodierschema vor.

Zum hedonischen Nutzen zählen Kriterien wie Spaß und Freude, während der utilitaristische Nutzen insb. die Informationsübermittlung und sonstige nützliche Aspekte umfasst. Die utilitaristischen und hedonischen Nutzenkomponenten wurden jeweils auf einer Skala von 0 bis 10 eingestuft. Manche MAR-Apps zielen auf eine genderspezifische Nutzergruppe ab. Bei Apps, die sich offensichtlich vorrangig an Frauen richten (z.B. bei Try-Ons für Make-up) wurde die Einstufung des Hauptkodierers durch das zusätzliche Urteil einer weiblichen Kodiererin validiert. Die deskriptiven Statistiken sowie die Interkorrelationen sind in Tab. 1 dargestellt. Bemerkenswert ist, dass die utilitaristischen und hedonischen Nutzenkomponenten negativ korreliert sind, während beide einen positiven Zusammenhang zu den User Ratings aufweisen.

Einige MAR-Apps können vorrangig indoor genutzt werden. Dies betrifft beispielsweise den Einsatz zuhause, in der Schule oder in Restaurants. Von den untersuchten Apps waren 31\% hierauf ausgerichtet, während $20 \%$ für eine OutdoorNutzung vorgesehen waren. Knapp die Hälfte der untersuchten Apps (49\%) kann sowohl indoor als auch outdoor genutzt werden (indoor/outdoor/beides: Entertainment und Social Media: 6/13/22; Bildung: 5/2/4, virtuelle Produktproben 13/0/13, nützliche Tools: 6/5/6, Information Layer: 1/0/4).

\section{Befunde}

Abb. 1 stellt die utilitaristischen und hedonischen Nutzenkomponenten verschiedener Typen von MAR-Apps vergleichend gegenüber. Varianzanalysen bestätigen, dass sich die fünf analysierten Typen hinsichtlich des utilitaristischen $(F(4)=13,091$, $p \leq 0,001)$ und hedonischen $(\mathrm{F}(4)=60,808, p \leq 0,001)$ Nutzens statistisch signifikant unterscheiden. Der Student-Newman-Keuls-Test ermittelt als Post-hoc-Analyse hinsichtlich des utilitaristischen Nutzens drei Gruppen, innerhalb derer sich keine 


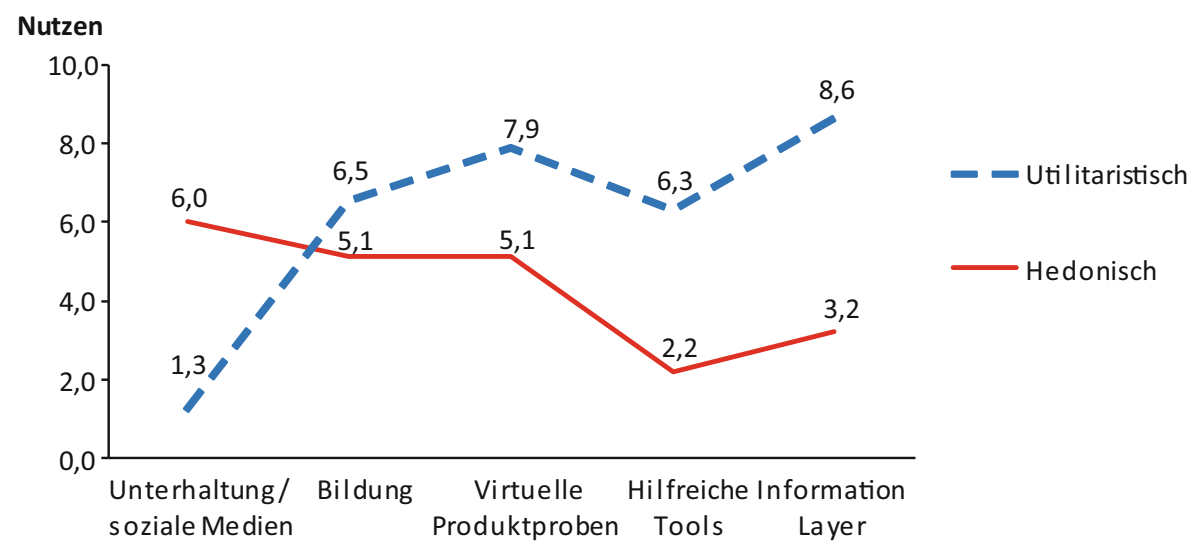

Abb. 1 Utilitaristischer und hedonischer Nutzen unterschiedlicher Typen von AR-Apps

statistisch signifikanten Unterschiede ergeben: (1) Information Layer und virtuelle Produktproben, (2) Bildung, hilfreiche Tools und virtuelle Produktproben und (3) Unterhaltung und Social Media. Bezüglich des hedonischen Nutzen ergeben sich die Gruppen (1) Unterhaltung und soziale Medien, virtuelle Produktproben und Bildung und (2) Information Layer und hilfreiche Tools. Offensichtlich bieten die MAR-Apps mit Information Layer und hilfreichen Tools hauptsächlich utilitaristischen Nutzen, während jene für Unterhaltung und soziale Medien vor allem hedonischen Nutzen erzeugen. Virtuelle Produktproben (die z. B. verschiedene virtual mirrors für Sonnenbrillen etc. beinhalten) und Bildungsangebote bieten meist sowohl utilitaristischen als auch hedonischen Nutzen.

Eine regressionsanalytische Auswertung mit dem PROCESS Makro (v3.5; Hayes 2017; alle Einflussgrößen zentriert) zeigt, dass sowohl die utilitaristische $(b=0,096$, $\mathrm{t}=4,508, p \leq 0,001)$ als auch die hedonische $(\mathrm{b}=0,077, \mathrm{t}=5,561, p \leq 0,001)$ Nutzenkomponente die Bewertungen der Nutzer:innen beeinflussen. Darüber hinaus kann ein Interaktionseffekt der beiden Einflussgrößen festgestellt werden: Der Effekt des hedonischen Nutzens einer MAR-App hängt vom Ausmaß des utilitaristischen Nutzens $a b(b=-0,014, t=-2,228, p \leq 0,05)$.

Die Spotlight-Analyse hilft, diese Interaktion zu qualifizieren: Der Effekt des hedonischen Nutzens auf die Nutzerbewertung ist statistisch signifikant bei einem geringen utilitaristischen Nutzen ( $-1 \mathrm{SD}: \mathrm{b}=0,162, \mathrm{t}=4,130, p \leq 0,001, \mathrm{CI} 95 \%$ [0,084; 0,239]). Bei einem hohen utilitaristischen Nutzen ist der Einfluss des hedonischen Nutzens nur noch marginal signifikant und deutlich schwächer (+1 SD: $b=0,052$, $\mathrm{t}=1,969, p=0,052$, CI $95 \%$ [-0,000; 0,104]) (siehe Abb. 2). Die Floodlight-Analyse ermittelt einen Johnson-Neyman-Signifikanzbereich bis zu einem Wert von 7,98 (unstandardisiert) des utilitaristischen Nutzens, der von mehr als zwei Drittel der MAR-Apps in der Stichprobe erreicht wird (68\%). Demnach spielt bei MAR-Apps, die den utilitaristischen Nutzen betonen, die hedonische Nutzenkomponente eine weniger bedeutende Rolle.

Um die Stabilität der Effekte zu prüfen, wurde ein Robustness Check mit folgenden Kontrollvariablen durchgeführt: Anzahl der Bewertungen $(b=0,022, t=0,448$, 


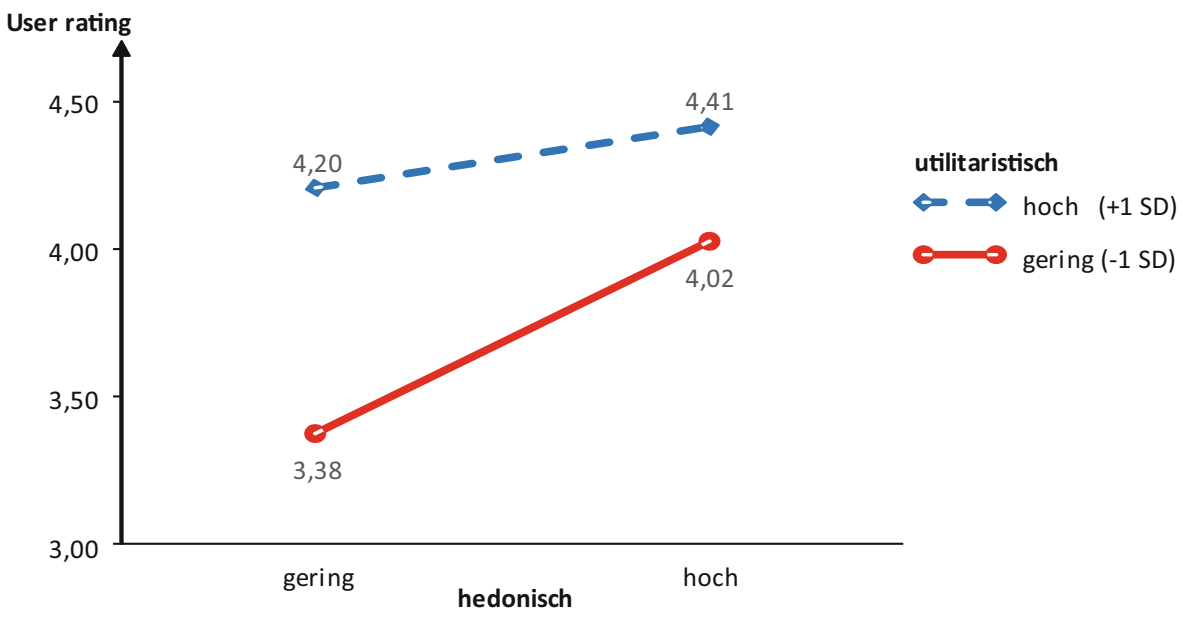

Abb. 2 Spotlight-Analyse: Effekt von utilitaristischem und hedonischem Nutzen auf User ratings

n. s.), Betriebssystem (Kodierung: 0 - Android, $1-\mathrm{iOS}, \mathrm{b}=0,278, \mathrm{t}=2,515, p \leq 0,05$ ) und Ort der Nutzung (dummy-kodiert, ,indoor“ als Baseline; ,outdoor“: $b=0,083$, $\mathrm{t}=0,743$, n.s.; ,indoor \& outdoor“: $\mathrm{b}=0,260, \mathrm{t}=1,844, p \leq 0,10)$. Die Befunde bleiben stabil (hedonisch: $\mathrm{b}=0,086, \mathrm{t}=3,926, p \leq 0,001$; utilitaristisch: $\mathrm{b}=0,078$, $\mathrm{t}=5,512, p \leq 0,001$; Interaktion: $\mathrm{b}=-0,013, \mathrm{t}=2,143, p \leq 0,05)$.

\section{Diskussion}

MAR-Apps für private Konsument:innen finden immer weitere Verbreitung und werden für mehr und mehr Einsatzgebiete entwickelt. Der vorliegende Beitrag analysierte 100 aktuelle MAR-Apps und kategorisierte sie entlang der Gruppen Unterhaltung und soziale Medien, Bildung, virtuelle Produktprobe, hilfreiche Tools und Information Layer. Erwartungskonform ist der Befund, dass MAR-Apps mit Information Layer und hilfreichen Tools hauptsächlich utilitaristischen Nutzen stiften, während MAR-Apps für Unterhaltung und soziale Medien hedonische Nutzenkomponenten haben. Interessanterweise bieten aber virtuelle Produktproben ebenso wie Bildungsangebote meist eine Kombination aus hohem utilitaristischen und hedonischen Nutzen. Sie können somit als Infotainment-MAR-Apps bezeichnet werden.

Im Fokus der Studie stand die Frage, wie diese Nutzenkomponenten die Nutzerbewertung in Form von User Ratings in den App-Stores prägen. Die Analyse zeigt, dass die User-Ratings gering sind, wenn weder hedonischer noch utilitaristischer Nutzen angeboten wird $(M=3,38)$. Bietet eine MAR nun entweder einen hedonischen Nutzen (4,02) oder einen utilitaristischen Nutzen $(4,20)$, steigt das User Rating spürbar an. Apps mit einer Kombination beider Nutzenarten performen insgesamt am besten (4,41). Die Untersuchung offenbart damit, dass die von Konsument:innen auf App-Stores abgegebenen Ratings der MAR-Apps zwar wie erwartet vom hedonischen und vom utilitaristischen Nutzen getrieben werden. Auffällig ist aber, 
dass die beiden Nutzenkomponenten negativ korrelieren. Es ergibt sich zudem ein bemerkenswerter Interaktionseffekt: Der hedonische Nutzen ist nur dann relevant, wenn der utilitaristische Nutzen gering ist. Bei geringem utilitaristischen Nutzen steigt die Nutzerbewertung proportional zum hedonischen Nutzen an. Bei einem hohen utilitaristischen Nutzen wird die Nutzerbewertung hierdurch definiert und der hedonische Nutzen schlägt nicht weiter merklich zu Buche (d.h. kein weiterer inkrementeller Nutzenbeitrag). Anders ausgedrückt: Bei hedonischem Nutzen kann ein weiterer utilitaristischer Nutzen das Rating zusätzlich steigern. Hat eine App bereits einen hohen utilitaristischen Nutzen, steigert eine Erhöhung des hedonischen Nutzens hingegen das User-Rating nicht weiter. Dies unterstreicht die herausragende Rolle des utilitaristischen Nutzens für die AR-Technologie.

Diese Befunde ergänzen die aktuelle Literatur insofern, als bisher der Beitrag der Nutzenkomponenten vor allem in Selbstauskünften von Nutzer:innen untersucht wurde und diese Untersuchungen auf einzelne Fallbeispiele von MAR-Apps beschränkt waren. Die vorliegende Untersuchung umspannt dagegen viele MAR-Apps und zieht als Kriterium eine objektive Angabe (User-Bewertungen auf den AppStores) heran.

Die Befunde sind für MAR-App-Entwickler:innen, Manager:innen wie auch für Forscher:innen zahlreicher Disziplinen, die sich mit AR-Anwendungen in ihren jeweiligen Feldern beschäftigen, relevant. Die Analyse zeigte, dass es bereits eine breite Anwendungspalette der Augmented Reality-Technologie für Apps auf mobilen Endgeräten für private Nutzer:innen gibt. Diese können für den Einsatz in Gebäuden, aber auch außerhalb und mobil genutzt werden, sodass eine Ausweitung auf zahlreiche weitere Anwendungsgebiete in Zukunft denkbar ist. Die Unterscheidung zwischen einem vorrangig hedonischen und/oder utilitaristischen Produktnutzen kann den Anbietern ganz generell bei der Entwicklung und Vermarktung helfen. MAR-Apps, die auf Unterhaltung (u. a. Games, Social Media) abzielen, können sich auf die hedonische Nutzenkomponente konzentrieren (=Toy). Auch MAR-Apps, die eine klare funktionale Unterstützung der Nutzer:innen durch Informationen oder sonstige Funktionalitäten liefern sollen (z. B. Produktinformationen, Navigationshilfen), werden gut bewertet, wenn sie auf die utilitaristische Komponente fokussieren (=Tool). Ein in Zukunft sicherlich stark wachsendes Anwendungsgebiet sind MARApps, die im stationären Einzelhandel eingesetzt werden können, um Kund:innen bei Bedarf und ggfs. personalisiert zusätzliche Produktinformationen zu übermitteln, die aufgrund des begrenzten Platzes auf den Produkten und dem Einkaufsregal nicht übermittelt werden können (Joerß et al. 2021). Die Kund:innen werden derartige Apps insbesondere nach dem funktionalen Wert der übermittelten Informationen bewerten. In vielen Anwendungsfällen, in denen die utilitaristische Komponente nur auf einem niedrigen oder mittleren Level ist, hilft dagegen eine gezielte Unterstützung durch hedonische Nutzenkomponenten. Dies trifft beispielsweise für Bildungsangebote zu, aber auch für virtuelle Produktproben für Shopping-Goods wie Schuhe, Bekleidung, Kosmetik oder Schmuck. Hier ist Infotainment gefragt (=Toy \& Tool). Für Marketer ist die Information, welche Nutzenkomponenten die MAR-App-Beurteilung (insb. die User-Ratings auf App-Stores) auch deshalb besonders wichtig, da dies für potenzielle weitere Nutzer:innen ein zentrales Merkmal darstellt, dass die Entscheidung für oder gegen den Download einer App beeinflusst. 
Schließlich sollte auch der aktuelle Entwicklungsstand der MAR-Apps betrachtet und ein Ausblick auf die zukünftige Bedeutung gewagt werden. Der Einsatz von AR stellt aktuell in vielen Anwendungsgebieten und aus der Sicht vieler Konsument:innen noch immer eine Innovation dar. Konsument:innen erleben den Einsatz von $\mathrm{AR}$ in Bereichen, in denen sie diese Funktion bislang noch nicht gesehen hatten, mit einer gewissen Neugierde und Freude, sodass der innovative Einsatz von AR per se die hedonische Nutzenkomponente erhöht (Beck und Crié 2018; Hopp und Gangadharbatla 2016; McLean und Wilson 2019; Yim et al. 2017). Dies gilt beispielsweise auch für die Bereitstellung von MAR-Einkaufshilfen im stationären Handeln, wo Produkte nur mit der Kamera des Smartphones erfasst werden müssen, um virtuell zusätzliche Produktinformationen zu erhalten (Joerß et al. 2021). Allerdings sind hier langfristig Wear-Out (Abnutzungseffekte) zu erwarten, wenn die Vertrautheit der Kund:innen mit diesen Tools wächst und wenn sich der Einsatz von AR ggfs. von einem Begeisterungsfaktor zu einem erwarteten Leistungsfaktor wandelt. Die Bedeutung der utilitaristischen Komponente wird somit in Zukunft vermutlich an Bedeutung gewinnen. Es steht zu erwarten, dass der professionelle und breite Einsatz von MAR-Apps zukünftig eine Verschiebung der Relevanz der hedonischen hin zur utilitiaristischen Nutzenkomponente bedeuten könnte.

Funding Open Access funding enabled and organized by Projekt DEAL.

Open Access Dieser Artikel wird unter der Creative Commons Namensnennung 4.0 International Lizenz veröffentlicht, welche die Nutzung, Vervielfältigung, Bearbeitung, Verbreitung und Wiedergabe in jeglichem Medium und Format erlaubt, sofern Sie den/die ursprünglichen Autor(en) und die Quelle ordnungsgemäß nennen, einen Link zur Creative Commons Lizenz beifügen und angeben, ob Änderungen vorgenommen wurden.

Die in diesem Artikel enthaltenen Bilder und sonstiges Drittmaterial unterliegen ebenfalls der genannten Creative Commons Lizenz, sofern sich aus der Abbildungslegende nichts anderes ergibt. Sofern das betreffende Material nicht unter der genannten Creative Commons Lizenz steht und die betreffende Handlung nicht nach gesetzlichen Vorschriften erlaubt ist, ist für die oben aufgeführten Weiterverwendungen des Materials die Einwilligung des jeweiligen Rechteinhabers einzuholen.

Weitere Details zur Lizenz entnehmen Sie bitte der Lizenzinformation auf http://creativecommons.org/ licenses/by/4.0/deed.de.

\section{Literatur}

Aluri A (2017) Mobile augmented reality (MAR) game as a travel guide: insights from Pokémon GO. J Hosp Tour Technol 8(1):55-72

Azuma RT, Baillot Y, Behringer R, Feiner S, Julier S, MacIntyre B (2001) Recent advances in augmented reality. Naval Research Lab, Washington, DC

Babin BJ, Darden WR, Griffin M (1994) Work and/or fun: measuring hedonic and utilitarian shopping value. J Consumer Res 20(4):644-656

Baek TH, Yoo CY, Yoon S (2018) Augment yourself through virtual mirror: the impact of self-viewing and narcissism on consumer responses. Int J Advert 37(3):421-439

Beck M, Crié D (2018) I virtually try it... I want it! Virtual fitting room: a tool to increase on-line and offline exploratory behavior, patronage and purchase intentions. J Retail Consumer Serv 40:279-286

Berryman DR (2012) Augmented reality: a review. Med Ref Serv Q 31(2):212-218

Botella CM, Lizandra MCJ, Baños RM, Raya MA, Guillén V, Rey B (2005) Mixing realities? An application of augmented reality for the treatment of cockroach phobia. Cyberpsychol Behav 8(2):162-171 
Bower M, Howe C, McCredie N, Robinson A, Grover D (2014) Augmented reality in education-cases, places and potentials. EMI Educ Media Int 51(1):1-15

Brito PQ, Stoyanova J (2018) Marker versus markerless augmented reality. Which has more impact on users? Int J Human Comput Interact 34:819-833

Carmigniani J, Furht B, Anisetti M, Ceravolo P, Damiani E, Ivkovic M (2011) Augmented reality technologies, systems and applications. Multimed Tools Appl 51(1):341-377

Chae HJ, Hwang JI, Seo J (2018) Wall-based space manipulation technique for efficient placement of distant objects in augmented reality. Proceedings of the 31st Annual ACM Symposium on User Interface Software and Technology, S 45-52

Childers TL, Carr CL, Peck J, Carson S (2001) Hedonic and utilitarian motivations for online retail shopping behavior. J Retail 77(4):511-535

Chung N, Lee H, Kim JY, Koo C (2018) The role of augmented reality for experience-influenced environments: the case of cultural heritage tourism in korea. J Travel Res 57(5):627-643

Dacko SG (2017) Enabling smart retail settings via mobile augmented reality shopping apps. Technol Forecast Soc Change 124:243-256

Davis FD (1989) Perceived usefulness, perceived ease of use, and user acceptance of information technology. MISQ 13(3):319-340

Dhar R, Wertenbroch K (2000) Consumer choice between hedonic and utilitarian goods. J Mark Res 37(1):60-71

Di Serio A, Ibáñez MB, Kloos CD (2013) Impact of an augmented reality system on students' motivation for a visual art course. Comput Educ 68:586-596

Dieck TMC, Jung T (2018) A theoretical model of mobile augmented reality acceptance in urban heritage tourism. Curr Issues Tour 21(2):154-174

Flavián C, Ibáñez-Sánchez S, Orús C (2019) The impact of virtual, augmented and mixed reality technologies on the customer experience. J Bus Res 100:547-560

Forbes (2020) The top 10 technology trends in retail: how tech will transform shopping in 2020. https:// www.forbes.com/sites/bernardmarr/2019/11/25/the-top-10-technology-trends-in-retail-how-techwill-transform-shopping-in-2020/?sh=6ba3a3174e03. Zugegriffen: 6. Dez. 2021

Hamari J, Malik A, Koski J, Johri A (2019) Uses and gratifications of pokémon go: why do people play mobile location-based augmented reality games? Int J Human Comput Interact 35(9):804-819

Harley JM, Poitras EG, Jarrell A, Duffy MC, Lajoie SP (2016) Comparing virtual and location-based augmented reality mobile learning: emotions and learning outcomes. Educ Technol Res Dev 64(3):359-388

Hayes AF (2017) Introduction to mediation, moderation, and conditional process analysis: a regressionbased approach, 2. Aufl. publications, Guilford

Heijden H van der (2004) User acceptance of hedonic information systems. MISQ 28(4):695-704

Heller J, Chylinski M, de Ruyter K, Mahr D, Keeling DI (2019a) Let me imagine that for you: transforming the retail frontline through augmenting customer mental imagery ability. J Retail 95(2):94-114

Heller J, Chylinski M, de Ruyter K, Mahr D, Keeling DI (2019b) Touching the untouchable: exploring multi-sensory augmented reality in the context of online retailing. J Retail 95(4):219-234

Hilken T, de Ruyter K, Chylinski M, Mahr D, Keeling DI (2017) Augmenting the eye of the beholder: exploring the strategic potential of augmented reality to enhance online service experiences. J Acad Mark Sci 45(6):884-905

Hopp T, Gangadharbatla H (2016) Novelty effects in augmented reality advertising environments: the influence of exposure time and self-efficacy. J Curr Issues Res Advert 37(2):113-130

Huang T-L, Liao S (2015) A model of acceptance of augmented-reality interactive technology: the moderating role of cognitive innovativeness. Electron Commer Res 15(2):269-295

Javornik A (2016) 'It's an illusion, but it looks real!' consumer affective, cognitive and behavioural responses to augmented reality applications. J Mark Manag 32(9-10):987-1011

Joerß T, Hoffmann S, Mai R, Akbar P (2021) Digitalization as solution to environ-mental problems? When users rely on augmented reality-recommendation agents. J Bus Res 128:510-523

Kaasinen E, Schmalfuß F, Özturk C, Aromaa S, Boubekeur M, Heilala J, Heikkilä P, Kuula T, Liinasuo M, Mach S, Mehta R, Petäjä E, Walter T (2020) Empowering and engaging industrial workers with Operator 4.0 solutions. Comput Ind Eng 139:105678

Kim HC, Hyun MY (2016) Predicting the use of smartphone-based augmented reality (AR): does telepresence really help? Comput Human Behav 59:28-38

Kourouthanassis P, Boletsis C, Bardaki C, Chasanidou D (2015) Tourists responses to mobile augmented reality travel guides: the role of emotions on adoption behavior. Pervasive Mob Comput 18:71-87 
Kytö M, Ens B, Piumsomboon T, Lee GA, Billinghurst M (2018) Pinpointing: precise head- and eye-based target selection for augmented reality. Proceedings of the $2018 \mathrm{CHI}$ Conference on Human Factors in Computing Systems, S 1-14

Masood T, Egger J (2019) Augmented reality in support of industry 4.0. Implementation challenges and success factors. Robot Comput Integr Manuf 58:181-195

McLean G, Wilson A (2019) Shopping in the digital world: examining customer engagement through augmented reality mobile applications. Comput Human Behav 101(8):210-224

Milgram P, Kishino F (1994) A taxonomy of mixed reality visual displays. IEICE Trans Inf Syst 77(12):1321-1329

Olsson T, Lagerstam E, Kärkkäinen T, Väänänen-Vainio-Mattila K (2013) Expected user experience of mobile augmented reality services: a user study in the context of shopping centres. Pers Ubiquit Comput 17(2):287-304

Pantano E, Rese A, Baier D (2017) Enhancing the online decision-making process by using augmented reality: a two country comparison of youth markets. J Retail Consumer Serv 38:81-95

Plotkina D, Saurel H (2019) Me or just like me? The role of virtual try-on and physical ap-pearance in apparel M-retailing. J Retail Consumer Serv 51:362-377

Rauschnabel PA (2018) Virtually enhancing the real world with holograms: an exploration of expected gratifications of using augmented reality smart glasses. Psychol Mark 35(8):557-572

Rauschnabel PA, Rossmann A, Dieck TMC (2017) An adoption framework for mobile augmented reality games: the case of Pokémon Go. Comput Human Behav 76:276-286

Rauschnabel PA, He J, Ro YK (2018) Antecedents to the adoption of augmented reality smart glasses: a closer look at privacy risks. J Bus Res 92(11):374-384

Rauschnabel PA, Felix R, Hinsch C (2019) Augmented reality marketing: how mobile AR-apps can improve brands through inspiration. J Retail Consumer Serv 49:43-53

Rese A, Baier D, Geyer-Schulz A, Schreiber S (2017) How augmented reality apps are accepted by consumers: a comparative analysis using scales and opinions. Technol Forecast Soc Change 124:306-319

Ruggiero TE (2000) Uses and gratifications theory in the 21st century. Mass Commun Soc 3(1):3-37

Samsung Business Insights (2020) 5 retail technology trends to watch in 2020. https://insights.samsung. com/2020/01/03/5-retail-technology-trends-to-watch-in-2020/. Zugegriffen: 15. Juni 2021

Smink AR, Frowijn S, van Reijmersdal EA, van Noort G, Neijens PC (2019) Try online before you buy: how does shopping with augmented reality affect brand responses and personal data disclosure. Electron Commer Res Appl 35:100854

Spreer P, Kallweit K (2014) Augmented reality in retail: assessing the acceptance and potential for multimedia product presentation at the poS. Trans Mark Res 1(1):20-35

Venkatesh V, Morris MG, Davis GB, Davis FD (2003) User acceptance of information technology: toward a unified view. MISQ 27(3):425-478

vXchange (2020) Top 7 augmented reality statistics for 2020. https://www.vxchnge.com/blog/augmentedreality-statistics. Zugegriffen: 15. Juni 2021

Vynz Research (2020) Augmented reality and virtual reality market. https://www.vynzresearch.com/ictmedia/augmented-reality-and-virtual-reality-market. Zugegriffen: 20. Aug.2021

Vávra P, Roman J, Zonča P, Ihnát P, Němec M, El-Gendi A (2017) Recent development of augmented reality in surgery: a review. J Healthc Eng. https://doi.org/10.1155/2017/4574172

Yang CC, Liu D (2017) Motives matter: motives for playing Pokémon Go and implications for well-being. Cyberpsychol Behav Soc Netw 20(1):52-57

Yaoyuneyong G, Foster J, Johnson E, Johnson D (2016) Augmented reality marketing: consumer preferences and attitudes toward hypermedia print ads. J Interact Advert 16(1):16-30

Yim MYC, Chu SC, Sauer PL (2017) Is augmented reality technology an effective tool for e-commerce? An interactivity and vividness perspective. J Interactive Mark 39:89-103

Zhou F, Duh HBL, Billinghurst M (2008) Trends in augmented reality tracking, interaction and display: a review of ten years of ISMAR. Proceedings of the 7th IEEE/ACM International Symposium on Mixed and Augmented Reality. IEEE Computer Society, S 193-202 